\title{
Suicide in dementia: 9-year national clinical survey in England and Wales
}

Nitin Purandare, Richard C. Oude Voshaar, Cathryn Rodway, Harriet Bickley, Alistair Burns and Nav Kapur

\section{Background}

Knowledge of suicide in people with dementia is limited to small case series.

\section{Aims}

To describe behavioural, clinical and care characteristics of people with dementia who died by suicide.

\section{Method}

All dementia cases $(n=118)$ from a 9-year national clinical survey of suicides in England and Wales ( $n=11512$ ) were compared with age- and gender-matched non-dementia cases (control group) ( $n=492)$ by conditional logistic regression.

\section{Results}

The most common method of suicide in patients with dementia was self-poisoning, followed by drowning and hanging, the latter being less frequent than in controls. In contrast to controls, significantly fewer suicides occurred within 1 year of diagnosis in patients with dementia. Patients with dementia were also less likely to have a history of self-harm, psychiatric symptoms and previous psychiatric admissions.

\section{Conclusions}

Known indicators of suicide risk are found less frequently in dementia suicide cases than non-dementia suicide cases. Further research should clarify whether suicide in dementia is a response to worsening dementia or an underappreciation of psychiatric symptoms by clinicians.

\section{Declaration on interest}

None. Funding detailed in Acknowledgements.
Prevention of suicide is a priority for health services. ${ }^{1}$ Historically, older age has been shown to be a risk factor for suicide. ${ }^{2,3}$ Over the past 15 years, suicide rates have fallen in some high-income countries, particularly in older age groups. ${ }^{4,5}$ However, the world population is aging and the study of suicide in older age remains important. Previous research suggests that suicide in older people is associated with depression, comorbid physical illnesses and substance misuse. . $^{2,5}$

Dementia is one of the major healthcare problems in later life, affecting 24 million people worldwide. ${ }^{7}$ Although high rates of psychiatric symptoms during the course of illness have been reported, ${ }^{8}$ the risk of suicide in people with dementia is generally considered low. ${ }^{9,10}$ Empirical data on suicide in dementia are scarce and primarily based on case reports (e.g. Rhode et $a l^{11}$ ). In a recent summary of those case reports, it has been suggested that early stage of dementia, male gender, high educational level, professional occupation, retention of insight, depressive features post-dating the onset of dementia, suicidal ideation and access to firearms might be risk factors for completed suicide in dementia. ${ }^{9}$ Furthermore, some studies have reported significantly more Alzheimer's pathology in the brains of older suicide victims compared with age- and gender-matched controls who died naturally, ${ }^{12}$ although findings have not been consistent. ${ }^{13}$

The National Confidential Inquiry into Suicide and Homicide by People with Mental Illness collects data on all those who die by suicide within 12 months of last contact with mental health services of the National Health Service (NHS) in the UK. ${ }^{14}$ It currently holds data on 11512 people aged 10 years and above who died by suicide in the period April 1996 to December 2004 and offers a unique opportunity to examine suicide in dementia in more detail. Our objectives were to describe the behavioural, clinical and care characteristics of patients with dementia who died by suicide, and to compare them with age- and gendermatched controls who died by suicide and who did not have clinical diagnosis of dementia.

\section{Methods}

The study was carried out as part of the National Confidential Inquiry into Suicide and Homicide by People with Mental Illness (the Inquiry), ${ }^{14}$ a complete national clinical sample of all people that had been in contact with mental health services in the 12 months prior to their death by suicide. The Inquiry has research ethics approval (South Manchester Research Ethics Committee ERP/96/2771) and approval under Section 60 of the Health and Social Care Act to process patient-identifiable data without consent. The data collection on suicides involved three stages: (a) collection of a comprehensive national sample, irrespective of mental health history; (b) identification of people in the sample who had been in contact with mental health services in the 12 months before death; and (c) collection of clinical data about these individuals. Data collection has been described in detail elsewhere, ${ }^{15}$ and is summarised below.

\section{Comprehensive national sample}

Information on all deaths in England and Wales receiving a suicide or an open verdict at coroner's inquest was obtained from the Office for National Statistics. The cases presented here consist of suicides registered by the Office for National Statistics from 1 April 1996 until 31 December 2004. In the first 3 years of the study, this information was cross-checked against equivalent data from health authorities in England and Wales; inconsistencies were rare. Open verdicts, recorded as deaths from undetermined external cause, are often reached in cases of suicide in the UK. Open verdicts are conventionally included in research on suicide and in official suicide statistics. ${ }^{16-18}$ In this study, we adopted this convention and included open (ICD-10 codes Y10-Y34, excluding Y33.9 (verdict pending) and Y87.2 (sequelae of events of 
undetermined intent)) as well as suicide (ICD-10 Codes X60-X84 and Y87.0) verdicts. ${ }^{19}$

\section{Identification of mental health service contact and collection of clinical data}

Identifying details on each individual who had died by suicide were submitted to the main hospital and community NHS trusts who provided mental health services to people living in the deceased's district of residence. When NHS records showed that contact had occurred in the 12 months before the suicide, the person became an 'Inquiry case'. For each Inquiry case, the consultant psychiatrist was sent a questionnaire and asked to complete it based on their knowledge of the patient, psychiatry case notes, and in discussion with other members of the mental health team. The questionnaire consisted of sections covering social/demographic characteristics, clinical history, details of suicide, aspects of care, details of final contact with mental health services and clinicians' views on suicide prevention. Clinicians were asked for an ICD-10 primary diagnosis and also any ICD-10 secondary diagnoses. ${ }^{19}$ The social and clinical items reflected many of the most frequently reported risk factors for suicide. The majority of items were factual but some (for example, adherence to treatment) were based on the judgements of clinicians.

\section{Identification of patients with dementia and selection of controls}

Based on the information provided by the responsible consultant psychiatrist, we identified all patients with an ICD-10 diagnosis of dementia. ${ }^{19}$ A total of 118 patients with dementia were identified. For each individual with dementia, we extracted four gendermatched suicide cases of patients without a diagnosis of dementia (controls) nearest in age to the dementia patients $(n=472)$. This selection was masked, i.e. we were unaware of the behavioural, clinical and care characteristics of these controls. All controls were individually matched for gender and 462/472 (98\%) for age within 2 years of the index case (3-year difference for seven cases, 5 -year difference for two cases and 6-year difference for one case).

\section{Statistical analysis}

All data are presented as absolute numbers and percentages within groups. If an item of information was not known for a case, the case was removed from the analysis of that item; the denominator in all estimates is therefore the number of valid cases for the item. Differences between cases and controls were tested by univariate conditional logistic regression analyses, using Intercooled Stata 9.0 for Windows. As the study was mainly exploratory, main findings are presented as odds ratios (OR) including 95\% confidence intervals (CI). Acknowledging heterogeneity in the cases of dementia, we also performed subgroup analyses. The following subgroups were defined: (a) patients with dementia aged 65 years and over; and (b) all dementia cases excluding those in which the individual had a comorbid diagnosis of alcohol dependence and/ or schizophrenia and other delusional disorders. Dementia associated with alcohol dependence or schizophrenia and related disorders is thought to be qualitatively different from dementia in older people caused by neurodegenerative and/or cerebrovascular processes (e.g. Alzheimer's disease, vascular dementia and dementia with Lewy bodies). ${ }^{20}$ Patients with dementia under the age of 65 years were not selected for subgroup analyses as we anticipated small numbers.

\section{Results}

Over the 9-year study period, the Inquiry received notifications of 45012 deaths by suicide, including 30794 (68\%) cases in which the coroner's verdict was suicide and 14218 (32\%) open verdicts or deaths from undetermined cause. Of these, 11733 (26.1\%) were confirmed to have been in contact with mental health services in the year prior to death. Completed questionnaires were received for 11512 cases, a response rate of $98.1 \%$. In 11141 cases (97.9\% of the 11375 cases that included data on primary diagnosis), a psychiatric diagnosis was recorded. In 118 (1.1\%) of these cases, the individuals had received a clinical diagnosis of dementia from their psychiatrist and $72(61 \%)$ had received an open verdict. As expected this was a higher proportion of open verdicts than in the control sample $(n=131,28 \%)$.

\section{Behavioural, clinical and care characteristics of the dementia group}

The 118 patients who had died by suicide and had a clinical diagnosis of dementia had a median age of 75 years (range 32$95)$ and $63(53 \%)$ were male. The sample was predominantly White, including only $5(4 \%)$ individuals from an ethnic minority group. Furthermore, 36 (32\%) individuals were married and 58 (49\%) lived alone at the time of suicide.

Online Table DS1 describes behavioural, clinical and care characteristics of the dementia group as a whole, and by predefined subgroups. For 91 (80\%) of the group, the last contact with services was routine or non-urgent, and for 57 (50\%) it occurred in patients' homes. A care-coordinator was allocated in $84(81 \%)$ cases and $47(40 \%)$ individuals were on an enhanced care programme approach (a form of enhanced aftercare indicative of clinical complexity and involvement of at least two members of a multidisciplinary team with different professional backgrounds, usually a community psychiatry nurse and a psychiatrist). The time between last contact and the suicide was less than 1 week for 50 (44\%) individuals, and less than $24 \mathrm{~h}$ for $18(16 \%)$. A further $29(25 \%)$ individuals had their last contact between 1 and 4 weeks before suicide, 13 (11\%) between 5 and 13 weeks, and $23(20 \%)$ more than 3 months before. Evidence of psychiatric symptoms at the last contact was noted for 41 $(35 \%)$ individuals, most often depressive illness $(n=18,16 \%)$, hopelessness $(n=10,9 \%)$ and delusions or hallucinations $(n=8$, $7 \%)$. In four patients (4\%) suicidal ideation was recorded at the last contact. A deterioration in physical health was noted for 27 (24\%) patients.

A total of 26 individuals who had died by suicide and had a diagnosis of dementia were aged below 65 years, and half of this younger subgroup $(n=13,50 \%)$ had a history of alcohol and/or drug misuse, including $6(23 \%)$ with a diagnosis of alcohol dependence. As shown in online Table DS1, this subgroup had more involved psychiatric histories and received more intense psychiatric care.

\section{Method of suicide}

Similar to the control group, self-poisoning was the most common method of suicide in the dementia group, accounting for almost a third of deaths (Table 1). The substance used was highly variable, most often being analgesics ( $n=12$ : paracetamol, $n=4$; codeine-containing preparations and other opiate analgesics, $n=4$; other analgesics, $n=4)$, psychotropic drugs ( $n=4$, all different classes), or other poisons such as weed killer $(n=4)$. All other substances were used only once. After self-poisoning, drowning $(19 \%)$ and hanging $(17 \%)$ were the second and third most 


\begin{tabular}{|c|c|c|c|c|c|c|c|c|}
\hline \multirow[b]{3}{*}{ Cause of death } & \multirow{3}{*}{$\begin{array}{l}\text { Dementia group } \\
\qquad \begin{array}{c}(n=118)^{a} \\
n(\%)\end{array}\end{array}$} & \multirow{3}{*}{$\begin{array}{l}\text { Control group } \\
\qquad \begin{array}{c}(n=472)^{\mathrm{a}} \\
n(\%)\end{array}\end{array}$} & \multicolumn{6}{|c|}{ Dementia subgroup statistics ${ }^{b}$} \\
\hline & & & \multicolumn{2}{|c|}{ Main statistics } & \multicolumn{2}{|c|}{$\begin{array}{c}\text { Age }>65 \text { years } \\
(n=92)\end{array}$} & \multicolumn{2}{|c|}{$\begin{array}{l}\text { Excluding patients with } \\
\text { comorbid schizophrenia and } \\
\text { alcohol dependence }(n=90)\end{array}$} \\
\hline & & & OR $(95 \% \mathrm{Cl})$ & $P$ & OR $(95 \% \mathrm{Cl})$ & $P$ & OR $(95 \% \mathrm{Cl})$ & $P$ \\
\hline Self-poisoning & $30(28)$ & $153(33)$ & $0.88(0.54-1.42)$ & 0.60 & $0.59(0.32-1.09)$ & 0.091 & & \\
\hline Drowning & $21(19)$ & $54(12)$ & $1.55(0.90-2.69)$ & 0.12 & & & & \\
\hline Hanging & $19(17)$ & $142(30)$ & $0.45(0.26-0.78)$ & 0.005 & & & $0.55(0.30-1.02)$ & 0.058 \\
\hline $\begin{array}{l}\text { Jumping } \\
\text { (height/moving vehicle) }\end{array}$ & $9(8)$ & $26(6)$ & $0.90(0.43-1.86)$ & 0.77 & & & & \\
\hline Suffocation & $6(6)$ & $35(8)$ & $0.75(0.30-1.88)$ & 0.49 & & & & \\
\hline Cutting or stabbing & $5(5)$ & $12(3)$ & $1.51(0.54-4.25)$ & 0.43 & & & & \\
\hline Other ${ }^{\mathrm{c}}$ & $27(23)$ & $30(6)$ & & & & & & \\
\hline
\end{tabular}

common methods of suicide among patients in the dementia group. The main analysis showed that hanging was used significantly less often by those in the dementia group compared with controls. Three suicides in the dementia group were part of a suicide pact.

\section{Comparison with the non-dementia group}

Patients with dementia who died by suicide were compared with their matched controls on all variables presented in online Table DS1. All demographic characteristics were similar for those in the dementia group and their age- and gender-matched controls $(P>0.43)$. In Table 2, behavioural, clinical and care variables that were significantly different in patients with dementia and their controls are presented. With respect to psychiatric history, patients with dementia less often died by suicide within 1 year of diagnosis, were less often prescribed antidepressants, and were less likely to have a history of self-harm. Although the main analysis suggests that patients with dementia were more likely to have a history of violence ( $12 \%$ v. $6 \%, P=0.018)$, the difference was quite small and was not replicated in the two subgroup analyses (Table 2). In line with these findings, patients with dementia had fewer psychiatric admissions and were also less likely to die by suicide while psychiatric in-patients. Significantly fewer psychiatric symptoms were reported during the last contact with a mental health service professional in patients with dementia compared

Table 2 Behavioural, clinical and characteristics of patients with dementia that differed significantly from their controls at time of suicide

\begin{tabular}{|c|c|c|c|c|c|c|c|c|}
\hline \multirow[b]{3}{*}{ Cause of death } & \multirow{3}{*}{$\begin{array}{l}\text { All dementia cases } \\
\qquad(n=118)^{\mathrm{a}} n(\%)\end{array}$} & \multirow{3}{*}{$\begin{array}{c}\text { Controls }(n=472)^{\mathrm{a}} \\
n(\%)\end{array}$} & \multicolumn{6}{|c|}{ Dementia subgroup statistics ${ }^{b}$} \\
\hline & & & \multicolumn{2}{|c|}{ Main statistics } & \multicolumn{2}{|c|}{$\begin{array}{c}\text { Age }>65 \text { years } \\
(n=92)\end{array}$} & \multicolumn{2}{|c|}{$\begin{array}{l}\text { Excluding patients with } \\
\text { comorbid schizophrenia and } \\
\text { alcohol dependence }(n=90)\end{array}$} \\
\hline & & & OR $(95 \% \mathrm{Cl})$ & $P$ & OR $(95 \% \mathrm{Cl})$ & $P$ & OR $(95 \% \mathrm{Cl})$ & $P$ \\
\hline $\begin{array}{l}\text { Psychiatric history } \\
\text { Duration of primary } \\
\text { diagnosis }<1 \text { year } \\
\text { Duration of primary }\end{array}$ & $16(14)$ & $134(31)$ & $0.34(0.20-0.61)$ & $<0.001$ & & & $0.31(0.16-0.59)$ & $<0.001$ \\
\hline $\begin{array}{l}\text { diagnosis }>5 \text { years } \\
\text { Previous psychiatric }\end{array}$ & $41(35)$ & $191(44)$ & $0.68(0.44-1.06)$ & 0.087 & $0.53(0.31-0.99)$ & 0.019 & $0.59(0.35-0.99)$ & 0.047 \\
\hline admissions & $63(54)$ & $337(72)$ & $0.43(0.27-0.67)$ & $<0.001$ & $0.35(0.21-0.57)$ & $<0.001$ & $0.35(0.21-0.58)$ & $<0.001$ \\
\hline History of self-harm & $50(44)$ & $265(58)$ & $0.59(0.39-0.90)$ & 0.013 & $0.42(0.26-0.69)$ & $<0.001$ & $0.55(0.34-0.89)$ & 0.014 \\
\hline History of violence & $14(12)$ & $28(6)$ & $2.47(1.17-5.20)$ & 0.018 & & & & \\
\hline History of alcohol misuse & $22(19)$ & $75(16)$ & $1.14(0.66-1.96)$ & 0.64 & & & $0.29(0.10-0.83)$ & 0.021 \\
\hline \multicolumn{9}{|l|}{ Clinical care } \\
\hline In-patient at time of suicide & $7(6)$ & $56(12)$ & $0.41(0.18-0.93)$ & 0.033 & $0.31(0.11-0.89)$ & 0.030 & $0.33(0.11-0.96)$ & 0.042 \\
\hline $\begin{array}{l}\text { Antidepressant prescribed } \\
\text { Under care of old age }\end{array}$ & $37(47)$ & $280(71)$ & $0.36(0.22-0.60)$ & $<0.001$ & $0.26(0.15-0.46)$ & $<0.001$ & $0.39(0.22-0.68)$ & 0.001 \\
\hline psychiatry & $60(76)$ & $233(67)$ & $4.41(1.39-14.0)$ & 0.012 & $3.08(0.99-9.64)$ & 0.053 & $5.37(1.42-20.3)$ & 0.013 \\
\hline \multicolumn{9}{|l|}{ Last contact with services } \\
\hline Within 7 days of death & $50(44)$ & $260(55)$ & $0.55(0.37-0.84)$ & 0.005 & $0.44(0.27-0.70)$ & 0.001 & $0.54(0.33-0.87)$ & 0.012 \\
\hline noted & $41(35)$ & $269(57)$ & $0.59(0.39-0.90)$ & $<0.001$ & $0.40(0.25-0.65)$ & $<0.001$ & $0.41(0.25-0.66)$ & $<0.001$ \\
\hline
\end{tabular}


with their matched controls, of which emotional problems $(31 \%$ v. $16 \%, P=0.002)$ and depressive symptoms $(40 \%$ v. $16 \%$, $P<0.001)$ reached statistical significance. Life events within 3 months prior to suicide were evaluated systematically in 14 different categories (e.g. interpersonal problems with significant others, financial problems, victim of violence). The frequency of all different life events was similar in patients with dementia and controls, including 'own physical health problems' which was the most often reported life event in both groups $(15 \% v$. $17 \%, P=0.82$ ).

\section{Discussion}

This paper describes a complete national sample of people with dementia who died by suicide while in the care, or having recently been in the care, of mental health services in England and Wales, a geographical area including over 55 million citizens. These cases comprise only $1 \%$ (equivalent to 13.5 deaths per year) of the full 9 -year sample of people who died by suicide within 12 months of contact with mental health services. This number may be considered to be low given that about 550000 people in England and Wales are suffering from dementia; ${ }^{21}$ however, we only included individuals who had been seen by specialist mental health services in the 12 months before death. Over a fifth of suicide cases in the dementia group were aged below 65 years, an age group in which the prevalence of dementia is rare $(<0.5 \%)$ compared with people aged over 65 years (in whom the prevalence increases sharply from $1.5 \%$ in those aged $65-70$ years to $24.8 \%$ in those aged over 85 years). ${ }^{7}$ This finding indicates a high proportional risk of suicide in younger patients with dementia compared with older patients with dementia, although this might also suggest that younger patients with dementia are more likely to be in contact with specialist services than their older counterparts. Detailed inspection of this younger age group showed high levels of psychiatric comorbidity and long-standing psychiatric problems.

The methods of suicide used by patients with dementia were comparable to those used by controls. Moreover, we did not find differences with respect to the classic risk factors for suicide in later life, such as physical illness and life events. ${ }^{10}$ Suicide in dementia, however, was characterised by fewer behavioural and clinical indicators of suicide risk compared with controls in our study, as well as when compared with previous studies on older suicide victims in general. ${ }^{22}$ The individuals with dementia who died by suicide were significantly less likely to have a history of self-harm or a history of psychiatric admissions. At the last contact prior to suicide, psychiatric symptoms were less likely to be noted in the dementia group than in the control group. It may be that in people with dementia, the cognitions or concerns about the progression of dementia are more important determinants of suicide. Alternatively, psychiatric symptoms, especially depressive symptoms, may be underrecognised in patients with dementia or wrongly attributed to being core symptoms of dementia because of overlap between depression and dementia. ${ }^{23}$ This latter explanation is especially relevant as depressive illness is the most important risk factor for suicide in older adults, with a population attributable risk of up to $74 \%{ }^{24}$ In addition, depressive illness may negatively mediate the effect of physical illness. ${ }^{9}$

Interestingly, the time since diagnosis in the dementia group was more often between 1 and 5 years compared with patients who had other psychiatric diagnoses, which is in line with results of a retrospective study of suicide in dementia cases admitted to a psychiatric hospital. ${ }^{25}$ This time period corresponds to the first two-thirds of the disease process, with a median survival time of
10.5 years from onset and 5.7 years from diagnosis, ${ }^{26}$ which significantly decreases with advancing age. ${ }^{27}$ The predominance of suicide in the first half of the dementia process might be explained by significantly less interference of cognitive deficits with the planning and executing of the suicide act at this stage. ${ }^{28,29}$ Moreover, depressive symptoms, which may have been underestimated in our sample, predominate the initial stage of the dementia process, whereas the latter stages are more dominated by apathy. ${ }^{29,30}$ Importantly, our data show a very low prevalence of suicide in the first 12 months after dementia has been diagnosed. This finding may indicate that disclosure of diagnosis, although it might evoke suicidal thoughts, is not a risk factor as sometimes suggested. ${ }^{31}$ Our results are most consistent with the view that the risk for suicide is highest at the stage of the disease process where patients experience increasing difficulties in daily living activities due to their dementia, but still have a preservation of insight into ongoing and impending cognitive deterioration. ${ }^{32}$ This point of view contrasts with suicides in other psychiatric disorders in which lack of insight is generally a risk factor for suicide. ${ }^{15}$

\section{Study limitations}

Although the large size of the database used in this study and high absolute number of dementia suicide cases enhances its inferential value, the findings of this study need to be interpreted in the context of certain methodological issues. First, this study is a survey of the clinical circumstances preceding suicide, and we are unable to make causal inferences. A control group of patients with dementia who had not died by suicide would have been relevant for identification of suicide risk factors specific for dementia. Controls were mental health patients who died by suicide with a diagnosis other than dementia. Therefore, the differences we observed could simply be due to differences between diagnostic groups (rather than differences in suicide risk). As depression (and rarely other psychiatric illnesses) may precede dementia, it is possible that some of the controls may in fact have undiagnosed early dementia. ${ }^{33}$ This raises the possibility that that observed differences between dementia suicides and the control group of non-dementia suicides may be underestimated. Known risk factors for suicide in later life (i.e. male gender and older age, could not be examined because of matched analyses). ${ }^{10,34}$ Second, the generalisability of our findings is limited to patients with dementia in contact with mental health services, most likely patients with dementia with significant behavioural and psychological symptoms. Therefore, suicides in patients with 'uncomplicated' dementia or those who are not receiving treatments to enhance cognition may be under-represented in this sample. Such a bias towards patients with dementia with behavioral difficulties or psychiatric comorbidity make the findings in relation to less symptomatology and comorbidity more remarkable. Moreover, this may also explain the low number of ethnic minorities, as elderly suicide victims from ethnic minorities are less likely to be in contact with psychiatric services. ${ }^{35}$ Third, the clinicians who provided the information by filling out the study questionnaires were not masked to patient outcome and this may have affected their response to certain questions, for example, the preventability of suicide in their patient. However, this will not affect the comparison between cases and controls, and the accuracy of the Inquiry questionnaire has been shown to be good. ${ }^{14}$

We used the wider definition of suicide that included both suicide and deaths of undetermined intent. This is the standard definition that is used in official statistics and previous research. Excluding open verdicts may underestimate the number of actual suicide deaths by $50 \% .{ }^{18}$ However, the number of open verdicts 
was much higher in the dementia group than the control group in this study - why might this be? A study of all suicide and open verdicts over a 10-year period in one coroner's court found that the factors which most reliably distinguished suicide from open verdicts included method of death (hanging and death from car exhaust poisoning more common; drowning and falling from a height less common) and presence of a suicide note. A small study of deaths in individuals aged 65 years found that evidence of suicidal intent, psychiatric history and method of death distinguished suicide and open verdicts. ${ }^{36}$ In our study, the cases with dementia included a lower proportion of hanging deaths and a higher proportion of drowning deaths than controls. Those with dementia were less likely to have a significant psychiatric history. Individuals with dementia who died by suicide were also presumably less likely than those with other diagnosis who died by suicide to indicate suicidal intent or to write a suicide note, although we did not investigate these factors directly.

\section{Clinical implications}

The antecedents and clinical characteristics of patients with dementia who die by suicide differ from age- and gender-matched suicide victims with other psychiatric diagnoses. The low prevalence of suicide in dementia combined with fewer longstanding psychiatric problems, as well as fewer psychiatric symptoms just before death compared with controls, means that identifying people with dementia who are at risk of suicide remains a significant clinical challenge. Patients may be at particular risk 1-5 years after diagnosis; during this period, suicidal thoughts could be openly discussed with patients and their families. This may help improve underrecognition and undertreatment of psychiatric comorbidity such as depression.

\footnotetext{
Nitin Purandare, PhD, FRCPsych, University of Manchester, Psychiatry Research Group, School of Community Based Medicine, Manchester, UK; Richard C. Oude Voshaar, MD, MSC, PhD, University of Manchester, Psychiatry Research Group Voshaar, MD, MSC, PhD, University of Manchester, Psychiatry Research Group,
school of Community Based Medicine, Manchester, UK, and Radboud University School of Community Based Medicine, Manchester, UK, and Radboud University
Nijmegen Medical Centre, Department of Psychiatry, Nijmegen, The Netherlands; Cathryn Rodway, MSc, Harriet Bickley, MSC, University of Manchester, Centre fo Suicide Prevention, Manchester, UK; Alistair Burns, FRCPsych, University of Manchester, Psychiatry Research Group, School of Community Based Medicine, Manchester, UK; Nav Kapur, MD, FRCPsych, University of Manchester, Centre for Suicide Prevention, Manchester, UK.

Correspondence: Dr N. Purandare, Room 3.316, Psychiatry Research Group, School of Community Based Medicine, University of Manchester, University Place (3rd Floor East), Oxford Road, Manchester M13 9PL, UK. Email: nitin.purandare@manchester.ac.uk
}

First received 29 Jan 2007, final revision 24 Jun 2008, accepted 22 Jul 2008

\section{Acknowledgements}

R.C.O.V and N.K. had full access to all the data in the study and are responsible for the integrity of the data and the accuracy of the data analysis. The National Confidential Inquiry receives funding from the National Patient Safety Agency of the Department of Health in England and Wales, the Scottish Executive, and the Department of Health, Social Security and Public Services in Northern Ireland.

\section{References}

1 Department of Health. National suicide prevention strategy for England. TSO (The Stationery Office), 2002.

2 Conwell Y, Duberstein PR, Cox C, Hermann JH, Forbes NT, Caine ED. Relationships of age and axis I diagnoses in victims of completed suicide: a psychological autopsy study. Am J Psychiatry 1996; 153: 1001-8.

3 World Health Organization. Suicide prevention. WHO, 2005. (http:// www.who.int/mental_health/prevention/suicide/suicideprevent/en/)
4 National Statistics. Suicide rates in United Kingdom. Office for National Statistics, 2007. (http://www.statistics.gov.uk/statbase/ Product.asp?vlnk=13618)

5 Koponen HJ, Viilo K, Hakko H, Timonen M, Meyer-Rochow VB, Sarkioja T, Rasanen P. Rates and previous disease history in old age suicide. Int J Geriatr Psychiatry 2007; 22: 38-46.

6 Waern M. Alcohol dependence and misuse in elderly suicides. Alcohol Alcohol 2003; 38: 249-54.

7 Ferri CP, Prince M, Brayne C, Brodaty H, Fratiglioni L, Ganguli M, et al. Global prevalence of dementia: a Delphi consensus study. Lancet 2005; 366: 2112-7.

8 Aalten $\mathrm{P}$, De Vught ME, Jaspers $\mathrm{N}$, Jolles J, Verhey FR. The course of neuropsychiatric symptoms in dementia. Part I: findings from the two-year longitudinal Maasbed study. Int J Geriatr Psychiatry 2005; 20: 523-30.

9 Lim WS, Rubin EH, Coats M, Morris JC. Early-stage Alzheimer disease represents increased suicidal risk in relation to later stages. Alzheimer Dis Assoc Disord 2005; 19: 214-9.

10 Conwell Y, Duberstein PR, Caine ED. Risk factors for suicide in later life. Biol Psychiatry 2002; 52: 193-204.

11 Rhode K, Peskind ER, Raskind MA. Suicide in two patients with Alzheimer's disease. J Am Geriatr Soc 1995; 43: 187-9.

12 Rubio A, Vestner AL, Stewart JM, Forbes NT, Conwell Y, Cox C. Suicide and Alzheimer's pathology in the elderly: A case-control study. Biol Psychiatry 2001; 49: 137-45.

13 Peisah C, Snowdon J, Gorrie C, Kril J, Rodriguez M. Investigation of Alzheimer's disease-related pathology in community dwelling older subjects who committed suicide. J Affect Disord 2007; 99: 127-32.

14 Appleby L, Shaw J, Sherrat J, Amos T, Robinson J, McDonnell R. Safety first: Five-year Report of the National Confidential Inquiry into Suicide and Homicide by People with Mental IIIness. Department of Health, 2001.

15 Hunt IM, Kapur N, Robinson J, Shaw J, Flynn S, Bailey H, Meehan J, Bickley H, Burns J, Appleby L, Parsons R. Suicide within 12 months of mental health service contact in different age and diagnostic groups: national clinical survey. Br J Psychiatry 2006; 188: 135-42.

16 O'Donnell I, Farmer R. The limitations of official suicide statistics. $\mathrm{Br} J$ Psychiatry 1995; 166: 458-61.

17 Neeleman J, Wessely S. Changes in classification of suicide in England and Wales; time trends and associations with coroner's professional background Psychol Med 1997; 27: 467-72.

18 Linsley KR, Schapira K, Kelly TP. Open verdict $v$. suicide - importance to research. Br J Psychiatry 2001; 178: 465-8.

19 World Health Organization. The ICD-10 Classification of Mental and Behavioural Disorders. WHO, 1992.

20 Olde Rikkert MGM, van der Flier WM, de Leeuw FE, Verbeek M, Jansen RWMM, Verhey F, Scheltens P. Multiple diagnostic tests are needed to assess multiple causes of dementia. Arch Neurol 2006; 63: 144-6.

21 Cognitive function and dementia in six areas in England and Wales: the distribution of MMSE and prevalence of GMS organicity level in the MRC CFA Study. The Medical Research Council Cognitive Function and Ageing Study (MRC CFAS). Psychol Med 1998; 28: 319-35.

22 Heisel MJ. Suicide and its prevention among older adults. Can J Psychiatry 2006; 51: 143-54

23 Purandare N, Burns A, Craig S, Faragher B, Scott K. Depressive symptoms in patients with Alzheimer's disease. Int J Geriatr Psychiatry 2001; 16: 960-4.

24 Beautrais AL. A case control study of suicide and attempted suicide in older adults. Suicide Life Threat Behav 2002; 32: 1-9.

25 Barak $Y$, Aizenberg D. Suicide amongst Alzheimer's disease patients: a 10year survey. Dement Geriatr Cogn Disord 2002; 14: 101-3.

26 Waring SC, Doody RS, Pavlik VN, Massman PJ, Chan W. Survival among patients with dementia from a large multi-ethnic population. Alzheimer Dis Assoc Disord 2005; 19: 178-83.

27 Xie J, Brayne C, Matthews FE. Survival times in people with dementia: analysis from population based cohort study with 14 year follow-up. BMJ 2008; 336: 258-62.

28 Rubin EH, Veiel LL, Kinscherf DA, Morris JC, Storandt M. Clinically significant depressive symptoms and very mild to mild dementia of the Alzheimer type. Int J Geriatr Psychiatry 2001; 16: 694-701.

29 Ready RE, Ott BR, Grace J, Cahn-Weiner DA. Apathy and executive dysfunction in mild cognitive impairment and Alzheimer disease. Am J Geriatr Psychiatry 2003; 11: 222-8.

30 Migliorelli R, Teson A, Sabe L, Petracchi M, Leiguarda R, Starkstein SE. Prevalence and correlates of dysthymia and major depression among patients with Alzheimer's disease. Am J Psychiatry 1995; 152: 37-44. 
31 Turnbull Q, Wolf AMD, Holroyd S. Attitudes of elderly subjects toward 'truth telling' for the diagnosis of Alzheimer's disease. I Geriatr Psychiatry Neurol 2003; 16: 90-3.

32 Vogel A, Stokholm J, Gade A, Andersen BB, Hejl AM, Waldemar G. Awareness of deficits in mild cognitive impairment and Alzheimer's disease: do $\mathrm{MCl}$ patients have impaired insight? Dement Geriatr Cogn Disord 2004; 17: 181-7.

33 Ownby RL, Crocco E, Acevedo A, John V, Loewenstein D. Depression and risk for Alzheimer Disease. Systematic review, meta-analysis, and metaregression analysis, Arch Gen Psychiatry 2006; 63: 530-8.
34 O'Connell H, Chin AV, Cunningham C, Lawlor BA. Recent developments: suicide in older people. BMJ 2004; 329: 895-9.

35 Salib E, Green L. Gender in elderly suicide: analysis of coroners inquest of 200 cases of elderly suicide in Cheshier 1989-2001. Int J Geriatr Psychiatry 2003; 18: 1082-7

36 Salib E. Coroner's verdicts in the elderly: a suicide or an open verdict? Int J Geriatr Psychiatry 1997: 12: 481-3.

\section{Psychiatrists in 19th-century fiction}

\section{Post-Mortem Recollections of a Medical Lecturer (1836), Charles Lever}

\section{Fiona Subotsky}

Charles Lever was an Anglo-Irishman who trained in medicine at Trinity College, Dublin, and St Steven's Hospital, but turned to writing in order better to support his family and early gambling habit. This extraordinary tale is purportedly written by a medical lecturer who has had an upsetting morning with the death of a patient but then goes on to give a lecture on insanity. As additional stressors, he is late and finds that the lecture theatre is extremely crowded, that an eminent foreign physician is present, and that he has left behind his main notes. Despite doubts, pauses and even repetitions he begins to warm to his theme, indeed words begin to fall 'with ease and rapidity'; he makes his points boldly and clearly, and easily demolishes theories of the past. Then he begins to think of his childhood, and shows that every case of those who died insane had some characteristic of a previous childhood trait, and develops this idea. He becomes more and more excited, and then full of terror:

'A thought rushed like a meteor flash across my brain, and bursting forth into a loud laugh of hysteric passion, I cried - and I, and I - too, am a maniac ... a cry of horror burst through the room. I know no more'.

When he comes round, he is in bed, presumably having succumbed to the 'brain fever', common in Victorian novels as the result of extreme stress. He tries to respond to the doctor and sit up, but feels a clammy perspiration, hears a rushing sound, suffers convulsive spasms and falls back again. 'It is over at last', the doctor says.

None the less, consciousness returns, though he is unable to move or speak. He knows he is dead, and wonders if it might be a trance, but knows 'all too well the unerring signs of death'. He is placed in a coffin, and friends gather round. He reflects that the processes of death are slow and that maybe, by the power of will, he can revive. He therefore proceeds, in a methodically medical fashion, 'to think upon those nerves which preside over the action of the heart, their origin, their course, their distribution, their relation, their sympathies'. He wills action, but fails at first, and is full of terror that the coffin is about to be closed. However, a dear friend puts his hand on his heart. Our narrator responds, and breathes once more.

There was a peculiarly Victorian fear of being buried alive which was exploited by a number of writers - for instance, Edgar Allan Poe in his story The Premature Burial (1844). The verisimilitude of Lever's tale is unusually enhanced by being told in a well-informed medical way, and indeed apparently had a basis in the author's own experience of a severe illness. The tone and mood, however, are notably 'gothic', and intended to induce horror and terror, as is the description of the descent into apparent madness. All this is most unlike the soothing reports of 'near-death experiences' of our own day. 